\title{
NOVEL ROOM TEMPERATURE MICROFLUIDIC DEVICE FABRICATION: A HIGH-RESOLUTION, 3D PRINTING APPROACH USING ELECTROHYDRODYNAMIC JET PRINTING
}

\author{
Christopher Pannier ${ }^{2 *}$, Zhi Wang ${ }^{2}$, David Hoelzle ${ }^{3}$, and Kira Barton ${ }^{l}$ \\ ${ }^{1}$ University of Michigan, Ann Arbor, Michigan, USA \\ ${ }^{2}$ University of Notre Dame, Notre Dame, Indiana, USA \\ ${ }^{3}$ The Ohio State University, Columbus, Ohio, USA
}

\begin{abstract}
For the first time, electrohydrodynamic-jet printing is used to fully fabricate a microfluidic device, achieving higher resolution $(<20 \mu \mathrm{m}$ channel width) and increased build material diversity compared to fully fabricating microfluidic devices by inkjet printing. This process is conducted at room temperature, making possible the inclusion of temperature-sensitive build materials during fabrication. A prototypical resistive pulse sensor (Coulter counter) is designed and printed to demonstrate the process. In the sensor, printed electronics are leveraged in the form of chemically sintered silver ink. Microfluidic channels are defined by a printed water-soluble polymer channel mold that is later washed away. Channel profiles are measured during fabrication, and channel profile shaping is demonstrated using an alternative, UV curable, channel mold material.
\end{abstract}

\section{INTRODUCTION}

Microfluidic devices have emerged as critical chemical, biological, and clinical instruments. The conventional microfluidic device fabrication processes, photolithography and soft lithography, have limited prototyping and customization capabilities due to the requirement of generating a photomask or mold for each device geometry change. Additionally, conventional processes use hightemperatures that preclude the incorporation of temperaturesensitive biomaterials and chemistries in device construction. Additive manufacturing (AM) of microfluidic devices offers rapid design iteration for prototyping and customization, with reduced fixed costs $[1,2]$. The AM-fabricated microfluidic device resolution and build material diversity depend on the particular AM process. Recently, fully inkjet printed microfluidic devices have been demonstrated with $60 \mu \mathrm{m}$ minimum channel width [3]. The devices were built from SU-8 epoxy build material, and had a maximum process temperature of $150^{\circ} \mathrm{C}$. Fully $3 \mathrm{D}$ printing a microfluidic device at room temperature with higher viscosity materials at a higher resolution than inkjet printing would be a key enabler for advancing the potential of bioprinting microfluidic devices.

One candidate AM process for improved 3D printing of microfluidic devices is electrohydrodynamic-jet (abbreviated here as e-jet, although some authors use EHD-jet) printing [4], which has demonstrated sub-100 $\mathrm{nm}$ resolution capability [5], and 3 orders of magnitude larger build material viscosities than inkjet printing [6]. E-jet printed applications include electronic sensors, bioassays, and bio-sensors $[5,6]$. In contrast to other jet-based printing techniques, e-jet uses an electric field to initiate drop ejection. A high voltage, typically between $200 \mathrm{~V}$ and $2000 \mathrm{~V}$, is applied to a conductive microcapillary nozzle of tip diameter between $100 \mathrm{~nm}$ and $30 \mu \mathrm{m}$ held at a small standoff height, typically $10 \mu \mathrm{m}$ to $200 \mu \mathrm{m}$ above the substrate, as shown in the inset of Fig. 1. The resulting electric field focuses the liquid meniscus into a conical shape, termed a Taylor cone, from which build material is ejected.

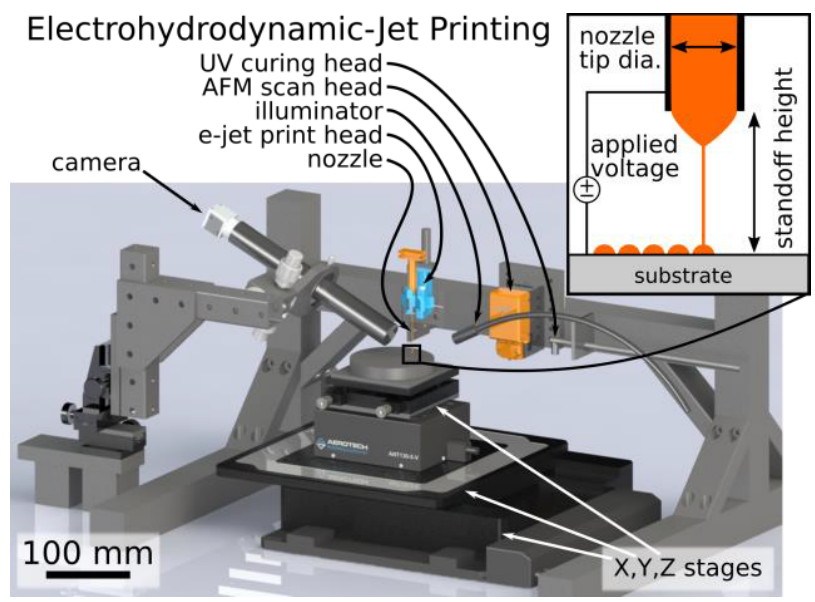

Figure 1: Rendering of the integrated e-jet/AFM system used for fully fabricating microfluidic devices. Inset is a schematic showing e-jet printing with key printing parameters.

Coordinated motion and jetting enable the printing of drops, lines, and layers onto a substrate. Build material is solidified by physical processes such as solvent evaporation, sintering, or UV curing. Successive deposition and solidification enable the fabrication of 3D microscale topographies [7, 8].

This work demonstrates the first use of e-jet printing to fully fabricate a microfluidic device entirely at room temperature. Full fabrication means that there are no spin coating or molding steps. The substrate is removed from the printer for a washing step at the end of the process. Printing, UV curing, and atomic force microscope (AFM) scanning is performed on the integrated ejet/AFM system housed at the University of Michigan [9], shown in Fig. 1. The use of e-jet printing greatly expands the available material diversity and resolution capability $(<20 \mu \mathrm{m})$ of jet printed microfluidic sensors.

The paper is organized as follows: The processes required to fully e-jet print a microfluidic device incorporating sensor electrodes and channels are selected and described in Process Design. The selection of a resistive pulse sensor (Coulter counter) as a demonstrative microfluidic device is described in Device Design. Printing steps and materials are reported in Experimental Methods. Achieved microfluidic channel geometry, improved channel resolution, and channel profile shaping are demonstrated in Results and Discussion. Finally conclusions and directions for future work are presented in Conclusion.

\section{PROCESS DESIGN}

\section{Room Temperature Processing}

Two challenging features for jet printing a microfluidic device are the covered microfluidic channel, which is a void supporting a 
channel wall, and the electrode, which must contact the microfluidic channel for sensing. The process design for forming channels and electrodes is key to fully realizing e-jet printing of a device at room temperature. Channels are produced by printing support material as a positive mold that is later dissolved. Electrodes are printed by leveraging a chemical sintering system from inkjet printed electronics.

A popular AM technique for producing voids is printing overhanging material with a printed dissolvable support material. A dissolvable support approach to printing microfluidic channels is taken in [3] by printing a build material solution of poly(methyl methacrylate) (PMMA) in anisole and dimethyl sulfoxide (DMSO). After printing, the solvent evaporates from the $40^{\circ} \mathrm{C}$ heated substrate and a subsequent $120^{\circ} \mathrm{C}$ hotplate solvent evaporation step. However, to achieve the room-temperature processing constraint proposed in this work, a dissolvable positive mold is printed using polyvinylpyrrolidone (PVP) in water and glycerol. In this approach, the bottom of the channel takes the shape of the already-printed layer. Next, the dissolvable material is printed to act as a positive mold for the channel. Subsequently, a non-dissolvable top layer is printed to cover the channel mold. The channel mold is dissolved in a solvent bath at the completion of the printed layers.

A common route to printed electronics, metal nanoparticle inks sintered after deposition, is used in this work. Thermally driven sintering at process temperatures of $80^{\circ} \mathrm{C}$ or higher is a standard sintering technique. Recently, room temperature chemical sintering has become commercially available for inkjet printable silver nanoparticle inks with instantly sintered sheet resistance within a factor of 4 of equivalent dimension copper trace sheet resistance [10]. Chemical sintering of these inks require a porous, chemical coating that is commercially available on polyethylene terephthalate (PET) film and glossy inkjet photo paper substrates. To meet the room temperature processing requirement, chemical sintering is used instead of thermal sintering in this work.

It is undesirable to locate a channel on a porous coating as leakage may result. Hence, an isolation layer is printed beneath the channel, except in the locations where electrode contact with a channel is desired. From bottom to top, the coated substrate, printed electrodes, isolation layer, channel mold, and top layer are shown for a channel that touches two electrodes in Fig. 2.
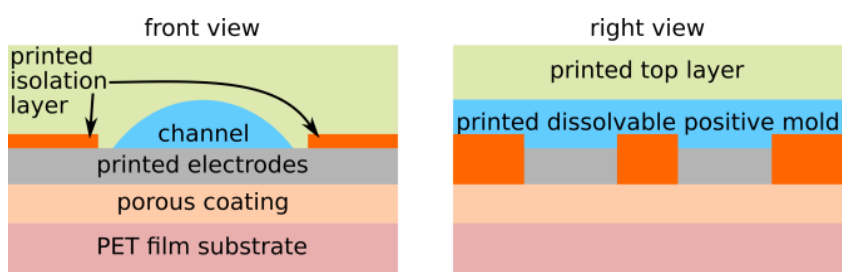

Figure 2: Front and right cross-section schematics of a resistive pulse sensor with e-jet printed electrodes, isolation layer, dissolvable channel mold, and top layer.

\section{Device Fabrication Steps}

Step a: E-jet print electrodes. First the substrate is loaded onto a vacuum chuck. The substrate will remain on the chuck throughout the fabrication until the final wash step. A nozzle containing the silver nanoparticle solution is loaded into the print head. The substrate $z$-axis position is adjusted to bring the nozzle to the appropriate standoff height relative to the substrate. The camera lens focal point is used for $x$ - and $y$-axis nozzle-to-substrate registration; nozzle and camera positions are adjusted to center the nozzle tip at the focal point of the camera lens. Coordinated motion and nozzle voltage is actuated from a script based on a sliced STL model.

Step b: E-jet print the isolation layer. After loading a nozzle containing UV curable build material for the isolation layer, the nozzle position is adjusted to center the nozzle tip in the camera field of view for registration with a standoff height set by the substrate $z$ axis stage motion. Printing is performed from a script. UV curing is performed after translating the substrate to the UV curing head.

Step c: E-jet print the channel mold. Replacing the nozzle with a nozzle containing dissolvable build material for the channel mold, the nozzle position is adjusted to bring the nozzle tip to the focal point of the camera for registration before setting the standoff height. Printing is performed from a script. UV curing is applied in the case of a UV curable channel mold build material. Optionally, AFM scanning of the channel mold is performed for channel metrology.

Step d: Print and then UV cure the top layer. The process in Step $b$ is then repeated using a different printing script.

Step e: Wash the device. The substrate is unchucked from its mount and immersed in a solvent bath to clear the channel. The five printing steps are labeled a-e in Fig. 3.

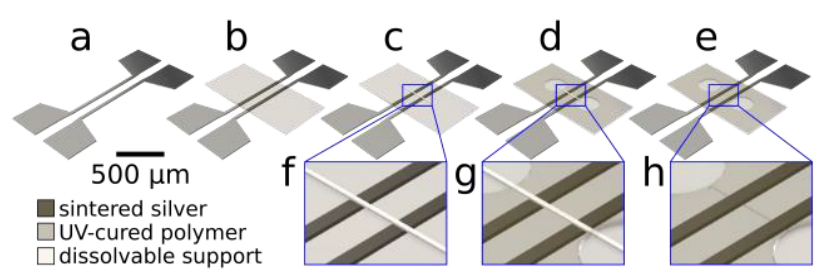

Figure 3: Steps to e-jet print a microfluidic resistive pulse sensor: (a) print electrodes onto substrate, (b) print isolation layer, (c) print dissolvable channel mold, (d) print top layer, and (e) wash support material in the channel. Detailed views of $(c-e)$ are shown in $(f-h)$.

\section{DEVICE DESIGN}

To demonstrate the e-jet printing of a microfluidic device, a single-channel resistive pulse sensor, a type of Coulter counter for particle counting and sizing, is designed and fabricated [11]. This sensor has a single $200 \mu \mathrm{m}$ long microfluidic channel with parallel electrodes beneath it to sense resistive pulses as a particle in an electrolyte solution flows past the electrodes. The electrodes are printed with contact pads on both sides of the device to verify printed trace conductivity. An isolation layer forms the base of the microfluidic channel. The channel is defined by a printed dissolvable positive mold. The top layer has ports for loading and unloading analyte liquid drops. To conserve build material and build time, the ports are designed for capillary driven flow into the device. Each port is a depression formed by printing a rim of build material in the top layer. A printed sensor is shown in Fig. 4. 


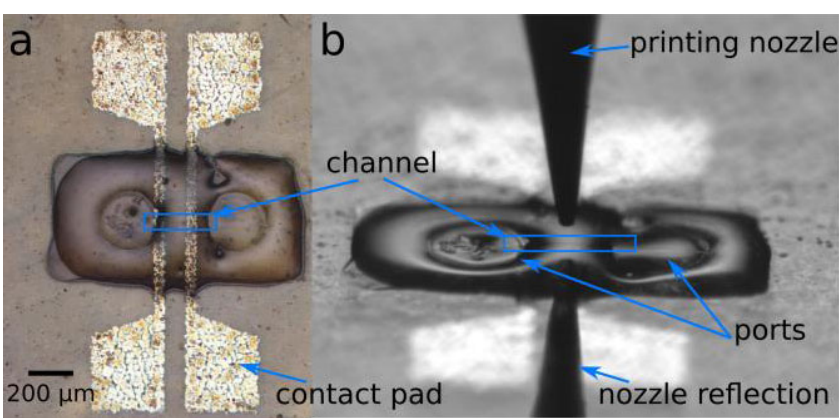

Figure 4. Top view (a) and side view (b) of an e-jet printed resistive pulse sensor just after fabrication step $d$. The $30 \mu \mathrm{m}$ tip diameter nozzle used to print the top layer is visible in (b).

\section{EXPERIMENTAL METHODS}

The electrode build material is a chemically sinterable silver nanoparticle ink (NBSIJ-MU01, Mitsubishi Imaging). Glycerol is added to the silver nanoparticle ink to avoid nozzle clogging from solvent evaporation. A $10 \mu \mathrm{m}$ nozzle tip diameter is used with the silver ink. The substrate is a flexible PET film substrate with porous chemical coating for instant chemical sintering of the ink (NB-TP3GU100, Mitsubishi Imaging). The isolation and top layer build material is a viscous (0.3 Pa-s), UV curable, thiol-ene based resin, Norland Optical Adhesive 81 (NOA81, Norland Products). A 30 $\mu \mathrm{m}$ nozzle tip diameter is used for increased NOA 81 deposition rate. NOA 81 is a commonly used build material for e-jet printing and other AM processes due to its fast $(<10$ s) UV curing, transparency, and solvent resistance [12]. The Young's modulus of NOA $81(\mathrm{O}(1) \mathrm{GPa})$, is comparable to that of SU-8 used in [3], and is intermediate between that of photolithographically-produced glass or silicon devices $(\mathrm{O}(100) \mathrm{GPa})$ and soft-lithographicallyproduced polydimethylsiloxane (PDMS) devices $(\mathrm{O}(0.001) \mathrm{GPa})$, making it a suitable material for microfluidic device construction.

For the channel mold build material, two dissolvable support materials are investigated. The first is an aqueous solution of the water-soluble polymer poly vinylpyrrolidone (PVP K15, average molecular weight 10,000 Da, Sigma-Aldrich) with added glycerol. A $10 \mu \mathrm{m}$ nozzle tip diameter is used for the PVP solution. The second is viscous (0.35 Pa-s), UV curable, acetone-debondable Norland Blocking Adhesive 107 (NBA107, Norland Products). A $10 \mu \mathrm{m}$ nozzle tip diameter is used for NBA107.

\section{RESULTS AND DISCUSSION}

A test device with $2.5 \mathrm{~mm}$ long channel is shown in Fig. 5c (with no electrodes). The PVP channel mold of the test device is scanned by the AFM during fabrication step $\mathrm{c}$ for channel metrology. An optical top view image is shown with the height profile inset in Fig. 5a. The channel width is $100 \mu \mathrm{m}$, and the channel height is $4.5 \mu \mathrm{m}$. The PVP channel mold forms an elliptical segment profile, similar to the results in [3]. To demonstrate flow in the channel, a solution of green food dye in water is loaded at the port and flows into the device by capillary action. The green dye with an air bubble in the channel is shown in Fig. 5 b.

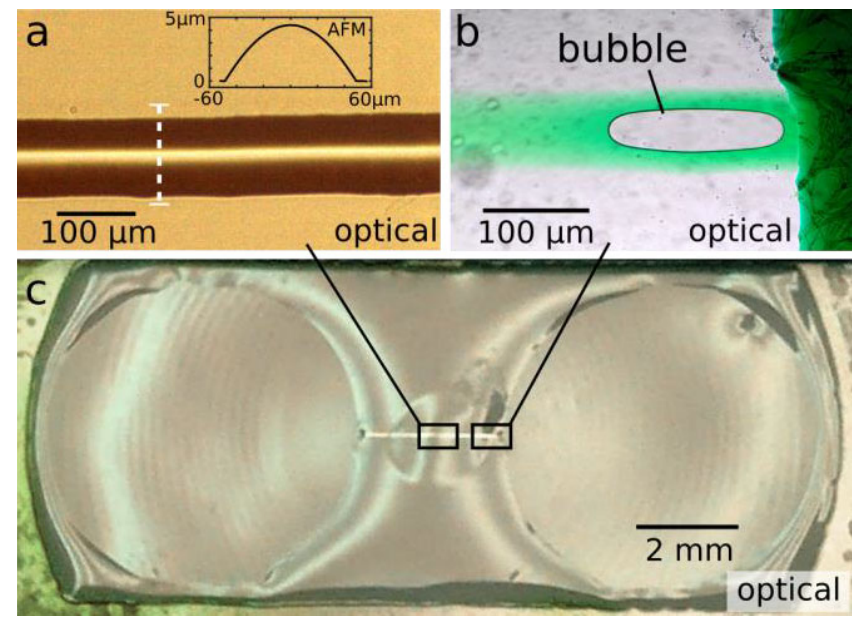

Figure 5. E-jet printed microfluidic channel test device without electrodes: (a) Top view of PVP channel mold after fabrication step c. Inset is a height profile of the channel collected by AFM scan along dashed line. (b) Top view of capillary filling of green dye into the finished device channel. (c) Top view of the completed device.

The influence of printing parameters on channel profile shape is studied for PVP channel molds. The number of printing passes is varied from 1 to 5 . The standoff height is varied between two values: a large standoff height of $70 \mu \mathrm{m}$, which gives a reduced deposition rate, and a reduced standoff high of $55 \mu \mathrm{m}$, which gives an increased deposition rate. The resulting channel images and profiles are shown in Fig. 6. The channel profiles are ellipsoidal caps with a variable contact angle. Channel widths below $30 \mu \mathrm{m}$ are achieved using the PVP channel mold.
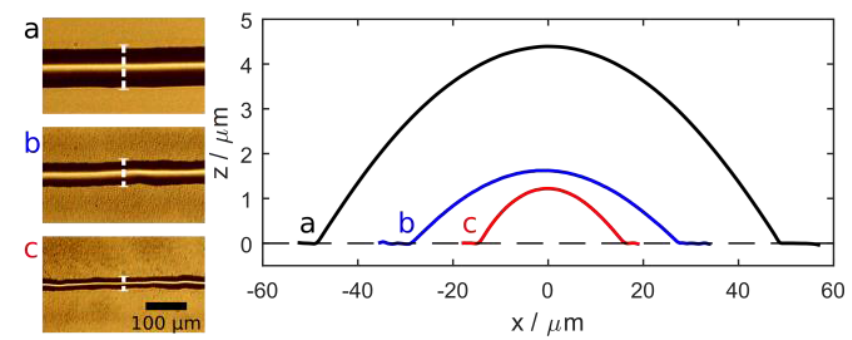

Figure 6. Top view microscope images and height profiles collected by AFM scans of three e-jet printed PVP channel molds. Varying printing parameters: (a) 5 passes at $45 \mu \mathrm{m}$ standoff height, (b) 3 passes at $70 \mu \mathrm{m}$ standoff height, and (c) 1 pass at $70 \mu \mathrm{m}$ standoff height, gave varying channel width, height, and contact angle.

Channel profile shaping is demonstrated using the UV curable NBA107 as the channel mold build material. Two variations of the printing procedure for multiple pass printing of NBA107 are investigated: one in which the deposited material is UV cured between each pass, and the alternative of UV curing only after the final pass has been deposited. The uncured NBA107 channel mold material cannot be AFM scanned because it is a liquid, so channel profiles are only available after UV curing to solidify the NBA107. When UV curing is applied at the end ( $a$ and d), an elliptical segment profile results, similar to the PVP results. UV curing applied 
between passes ( $b$ and $c$ ) results in a peaked profile with a pedestal, shown in Fig. 7.

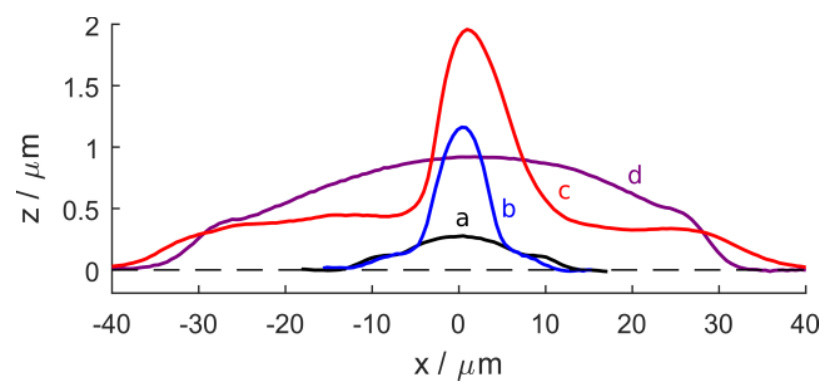

Figure 7. Height profiles collected by AFM scans of e-jet printed NBA107 channel molds printed as: (a) 1 pass (b) 4 passes, curing between passes, (c) 10 passes, curing between passes, (d) 10 passes, curing only after pass 10.

Channel widths below the $60 \mu \mathrm{m}$ inkjet minimum reported in [3] are readily achieved by e-jet printing the channel mold with either PVP $(<30 \mu \mathrm{m})$ or NBA107 $(<20 \mu \mathrm{m})$. Further reduction in ejet printed channel width is expected for smaller nozzles and printing parameter optimization.

\section{CONCLUSION}

This work demonstrates the first microfluidic device fully fabricated by the microscale additive manufacturing technique e-jet printing. Importantly, the fabrication process is conducted entirely at room temperature, with no spin coating or molding processes. Microfluidic channels are formed by printing water-soluble positive molds. Electrodes are printed using chemically sintered silver nanoparticle ink onto a chemically coated substrate. Whereas [3] uses thermally sintered silver nanoparticle ink for its electrodes and thermally crosslinked photoresist SU-8 for its isolation and top layers, the use of chemically sintered silver nanoparticle ink and UV adhesives in this work allows the device to be fabricated entirely at room temperature. Channel profiles are measured before printing the top layer onto the channel mold. The polymer channel mold formed by solvent evaporation assumes an ellipsoidal segment shape; however, channel profile shaping is demonstrated for a UV curable channel mold. This channel profile metrology technique offers the possibility of building up desired channel geometry in a controlled fashion through iterative printing and scanning steps. This demonstration of a fully e-jet printed microfluidic sensor at room temperature introduces e-jet printing as a powerful tool for high-resolution microfluidic device fabrication that enables the inclusion of temperature-sensitive materials.

Future areas to explore with this technique include methods to print chemically sintered electrodes in offset planes that are nonparallel to the substrate plane, as well as further demonstrations of build material diversity. In particular, future work aims to investigate e-jet printing of a microfluidic device constructed of thermally and chemically sensitive biomaterials that cannot be patterned conventionally, but are made possible using the presented room temperature e-jet printing technique.

\section{ACKNOWLEDGEMENTS}

This work was supported in part by NSF Awards CMMI1434660, CMMI-1434693, and GRFP-1256260. Travel support has been generously provided by the Transducer Research Foundation.

\section{REFERENCES}

[1] C.M.B. Ho, S.H. Ng, K.H.H. Li, Y.-J. Yoon, "3D Printed Microfluidics for Biological Applications”, Lab Chip, 15, 3627 (2015). doi.org/10.1039/c5lc00685f

[2] R.D. Sochol, E. Sweet, C.C. Glick, S. Venkatesh, et al., "3D Printed Microfluidic Circuitry via Multijet-Based Additive Manufacturing", Lab Chip, 16, $668 \quad$ (2016). doi.org/10.1039/c5lc01389e

[3] W. Su, B.S. Cook, Y. Fang, and M.M. Tentzeris, "Fully InkjetPrinted Microfluidics: A Solution to Low-Cost Rapid ThreeDimensional Microfluidics Fabrication with Numerous Electrical and Sensing Applications", Scientific Reports, 6, 35111 (2016). doi.org/10.1038/srep35111

[4] J.-U. Park, M. Hardy, S.J. Kang, K. Barton, et al., "HighResolution Electrodynamic Jet Printing", Nature Materials, 6, 782 (2007). doi.org/10.1038/nmat1974

[5] M.S. Onses, E. Sutanto, P.M. Ferreira, A.G. Alleyne, and J.A. Rogers, "Mechanisms, Capabilities, and Applications of HighResolution Electrohydrodynamic Jet Printing", Small, 11, 4237 (2015). doi.org/10.1002/smll.201500593

[6] C.P. Pannier, Z. Wang, D.J. Hoelzle, and K.L. Barton, "Electrohydrodynamic Jet Printing: A 3D Printing Technique for Sensor Fabrication," Technical Digest of the 2016 SolidState Sensor and Actuator Workshop, Hilton Head Isl., SC, 6/5-9/16, Transducer Research Foundation, Cleveland (2016), pp. $149-152$.

[7] Z. Wang, C. Pannier, K. Barton, D.J. Hoelzle, “Application of Robust Monotonically Convergent Spatial Iterative Learning Control to Microscale Additive Manufacturing”, Mechatronics (2018). Submitted.

[8] Z. Wang, P.M. Sammons, C.P. Pannier, K. Barton, and D.J. Hoelzle, "System Identification of a Discrete Repetitive Process Model for Electrohydrodynamic Jet Printing," Proc. of the 2018 American Control Conference, Milwaukee, WI, 6/2729/18, Institute of Electrical and Electronics Engineers, New York (2018). Accepted.

[9] C.P. Pannier, L. Ojeda, Z. Wang, D. Hoelzle, K. Barton, “An Electrohydrodynamic Jet Printer with Integrated Metrology", Mechatronics (2018). Accepted.

[10] Y. Kawahara, S. Hodges, B. S. Cook, C. Zhang, and G. D. Abowd, "Instant Inkjet Circuits: Lab-Based Inkjet Printing to Support Rapid Prototyping of UbiComp Devices", Proc. of the 2013 ACM International Joint Conference on Pervasive and Ubiquitous Computing, Zurich, Switzerland, 9/8-12/13, Association for Computing Machinery, New York (2013), pp. 363-372. doi.org/10.1145/2493432.2493486

[11] J. Zhe, A. Jagtiani, P. Dutta, J. Hu, and J. Carletta, "A Micromachined High Throughput Coulter Counter for Bioparticle Detection and Counting", J. Micromech. Microeng., 17, 304 (2007). doi.org/10.1088/0960$1317 / 17 / 2 / 017$

[12] K.W. Bong, J. Xu, J.-H. Kim, S.C. Chapin, M.S. Strano, K.K. Gleason, P.S. Doyle, "Non-Polydimethylsiloxane Devices for Oxygen-Free Flow Lithography", Nature Comm., 3, 805 (2012). doi.org/10.1038/ncomms 1800

\section{CONTACT}

*C.P. Pannier, PANNIER @ UMICH . edu 